\title{
Exact ground states of quantum spin-2 models on the hexagonal lattice
}

\author{
Marc André Ahrens * Andreas Schadschneider 1 and Johannes Zittart2: \\ Institute for Theoretical Physics, University of Cologne, 50937 Köln, Germany
}

(Dated: October 25, 2018)

\begin{abstract}
We construct exact non-trivial ground states of spin-2 quantum antiferromagnets on the hexagonal lattice. Using the optimum ground state approach we determine the ground state in different subspaces of a general spin-2 Hamiltonian consistent with some realistic symmetries. These states, which are not of simple product form, depend on two free parameters and can be shown to be only weakly degenerate. We find ground states with different types of magnetic order, i.e. a weak antiferromagnet with finite sublattice magnetization and a weak ferromagnet with ferrimagnetic order. For the latter it is argued that a quantum phase transition occurs within the solvable subspace.
\end{abstract}

PACS numbers: 75.10.Jm, 73.43.Nq, 75.25.+z

\section{INTRODUCTION}

Usually one faces the problem of finding the ground state and excitations of a given (many-body) Hamiltonian that is supposed to describe the physical problem of interest. The solution then allows, at least in principle, to determine other quantities, e.g. correlation functions. Usually an exact solution is not possible, especially in dimensions $d \geq 2$, and one has to rely on approximations (e.g. perturbation theory) or numerical methods.

Another approach does just the opposite ${ }^{1}$. It starts with a given ground state $\left|\psi_{0}\right\rangle$ and tries to find the corresponding Hamiltonian. This can be achieved by finding positive-semidefinite operators that annihilate $\left|\psi_{0}\right\rangle$. Then any Hamiltonian which is a sum of these operators has $\left|\psi_{0}\right\rangle$ as a ground state.

The optimum ground state (OGS) approach developed in 2,3 4 allows for a systematic construction of such ground states. For a given class of Hamiltonians one searches for subspaces of the space of interaction parameters where the determination of the ground state can be reduced to a local problem. OGS are characterized by the fact that they are simultaneously ground states of all local interactions contained in the Hamiltonian. It should be emphasized that despite this reduction to a local problem the properties of the ground state still depend on the structure of the lattice.

Generic examples are simple tensor product states like the fully polarized ferromagnet $|\uparrow \uparrow \cdots \uparrow\rangle$. However, the OGS approach is more flexible and also allows the construction of antiferromagnetic states that have a more complicated structure. This is possible if the ground state of the local Hamiltonian is degenerate. For onedimensional quantum spin chains the generic realization of OGS are matrix-product ground states 5.6 .7 . These have been studied systematically for spin- 1 [2, spin-3/2 [4] and spin-2 8] quantum spin chains revealing the existence of various phases, e.g. different types of Haldane phases or magnetically ordered states. Here all ground state properties, especially correlation functions, can be evaluated exactly in closed form. It should be mentioned that matrix-product states also play an important role for the DMRG-technique ${ }^{9.10 .11}$ which can be interpreted as variational method based on states of matrix-product form $12,13,14$.

However, the true power of the OGS approach is revealed by the application to spin models in higher dimensions $d \geq 2$. Technically this generalization is achieved by the vertex state model approach ${ }^{15}$. Here to each vertex of a vertex model a quantum state is assigned in addition to a Boltzmann weight. This allows for a very efficient bookkeeping and provides an elegant way of specifying the ground state. Ground state properties like correlation functions are then determined by the partition function of a classical vertex mode ${ }^{16}$ on the same lattice.

This approach has already been applied successfully to spin- $3 / 2$ systems on the hexagonal lattice $\frac{15}{}$ and spin2 systems on the square lattice $\frac{17}{7}$. These models can be considered as anisotropic generalizations of the valencebond-solid (VBS) model of Affleck et al $\stackrel{18}{18}$ since for a generic VBS state the spin $S$ and the coordination number $z$ of the lattice are related by $S=z / 2$.

An important point is the introduction of anisotropic interactions. This leads to whole families of solvable models and allows to study the parameter-dependence of the ground state properties, in contrast to the isotropic VBS-type models which are usually solvable only at isolated points. Indeed in both cases ${ }^{15.17}$ a quantum phase transition from a disordered to a Néel-ordered phase was found. As mentioned above the properties of these quantum-critical points are determined by the critical behaviour of a classical vertex model on the same lattice. A similar situation has recently been studied by Ardonne et al $\stackrel{19}{\underline{19}}$. They considered the quantum dimer model introduced by Rokhsar and Kivelson ${ }^{20}$. For this model the ground state can be determined exactly. It turns out to be an optimum ground state of a very simple form, namely an equal amplitude superposition of states connected by the dynamics. On a square lattice the Rokhsar-Kivelson model is critical. This allows also to study the influence of small perturbations on the critical behaviour ${ }^{19.21}$. For the Rokhsar-Kivelson model it turns out that perturbations can be relevant or irrelevant, depending on the lattice structure. 
The paper is organized as follows. In Sec. III we give a rather general introduction to the OGS approach. In Sec.III the OGS for the spin-2 case on a hexagonal lattice are determined. Here also the properties of these states are discussed qualitatively. Sec. IV contains concluding remarks and an outlook on future work.

\section{OPTIMUM GROUND STATES}

In the following we describe the basic idea of the OGS approach which will then be applied to spin- 2 models on the hexagonal lattice.

As mentioned before the OGS approach starts from a general class of Hamiltonians determined by the physical problem and its symmetries. First the most general Hamiltonian $\mathcal{H}$ with nearest neighbor interactions consistent with the following symmetries is constructed:

1. homogeneity in real space,

2. parity invariance (i.e. $h_{i j}=h_{j i}$ ),

3. rotational invariance in the $x y$-plane of spin space,

4. spin-flip invariance.

All of these requirements are quite natural for a twodimensional lattice. The Hamiltonian then has the general structure

$$
\mathcal{H}=\sum_{\langle i, j\rangle} h_{i j}
$$

with nearest neighbor sites $\langle i, j\rangle$.

Table I shows the most general basis vectors of the space of local interactions for spin $S=2$ that are consistent with the symmetries in terms of $S_{i}^{z}$-eigenstates

$$
S_{i}^{z}|m\rangle_{i}=m_{i}|m\rangle_{i} \quad \text { with } m \in\{0, \pm 1, \pm 2\}
$$

of the local spin-2 Hilbert space. It contains seven parameters $a_{1}, \ldots, a_{7}$ that correspond to rotations of the basis vectors in the respective subspaces that do not violate the symmetry requirements.

The general form of the local interaction $h_{i j}$ can then be expressed through the projectors on the symmetry-
TABLE I: The symmetry-consistent spin-2 basis states in the space of local interactions. Here $|m, \tilde{m}\rangle_{ \pm}$denotes the (anti-) symmetrization $|m, \tilde{m}\rangle_{ \pm}=|m, \tilde{m}\rangle \pm|\tilde{m}, m\rangle, \mu=m+\tilde{m}$ and $p= \pm 1$ for symmetric and antisymmetric states.

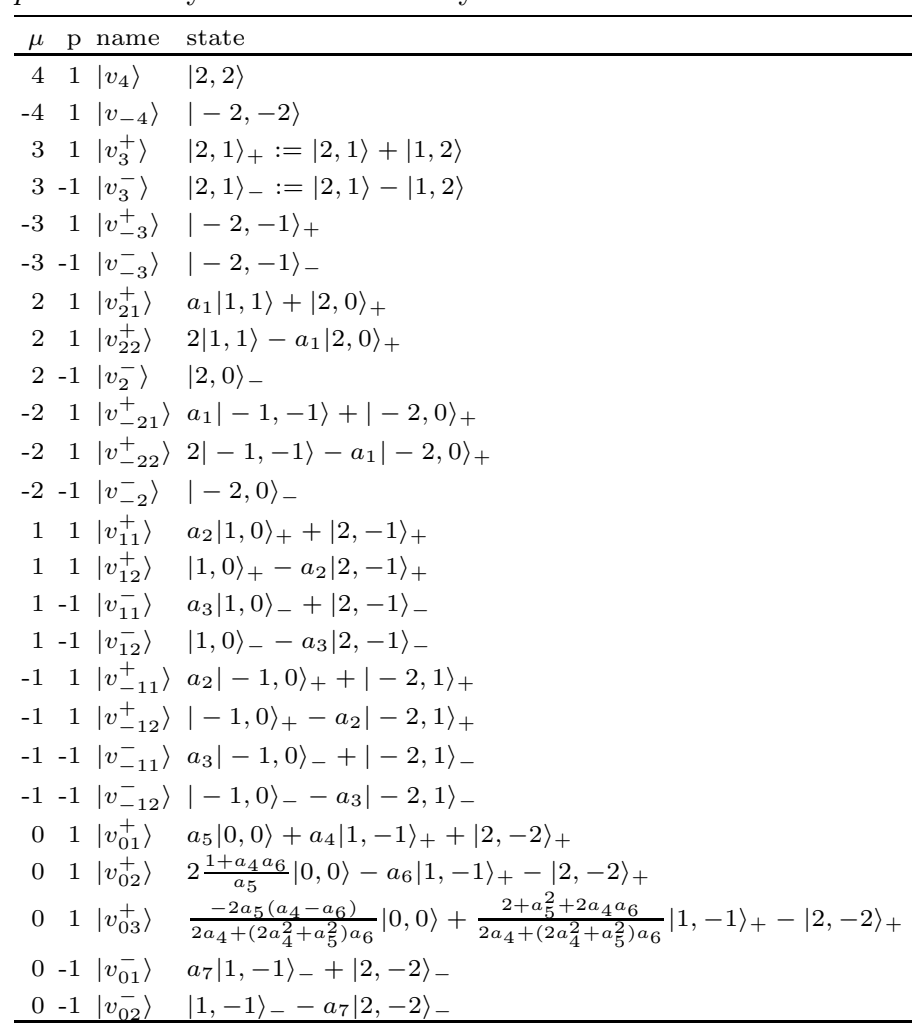

consistent basis vectors:

$$
\begin{aligned}
h_{i j}= & \lambda_{4}\left(\left|v_{4}\right\rangle\left\langle v_{4}|+| v_{-4}\right\rangle\left\langle v_{-4}\right|\right)+ \\
& \lambda_{3}^{+}\left(\left|v_{3}^{+}\right\rangle\left\langle v_{3}^{+}|+| v_{-3}^{+}\right\rangle\left\langle v_{-3}^{+}\right|\right)+ \\
& \lambda_{3}^{-}\left(\left|v_{3}^{-}\right\rangle\left\langle v_{3}^{-}|+| v_{-3}^{-}\right\rangle\left\langle v_{-3}^{-}\right|\right)+ \\
& \lambda_{21}^{+}\left(\left|v_{21}^{+}\right\rangle\left\langle v_{21}^{+}|+| v_{-21}^{+}\right\rangle\left\langle v_{-21}^{+}\right|\right)+ \\
& \lambda_{22}^{+}\left(\left|v_{22}^{+}\right\rangle\left\langle v_{22}^{+}|+| v_{-22}^{+}\right\rangle\left\langle v_{-22}^{+}\right|\right)+ \\
& \lambda_{2}^{-}\left(\left|v_{2}^{-}\right\rangle\left\langle v_{2}^{-}|+| v_{-2}^{-}\right\rangle\left\langle v_{-2}^{-}\right|\right)+ \\
& \lambda_{11}^{+}\left(\left|v_{11}^{+}\right\rangle\left\langle v_{11}^{+}|+| v_{-11}^{+}\right\rangle\left\langle v_{-11}^{+}\right|\right)+ \\
& \lambda_{12}^{+}\left(\left|v_{12}^{+}\right\rangle\left\langle v_{12}^{+}|+| v_{-12}^{+}\right\rangle\left\langle v_{-12}^{+}\right|\right)+ \\
& \lambda_{11}^{-}\left(\left|v_{11}^{-}\right\rangle\left\langle v_{11}^{-}|+| v_{-11}^{-}\right\rangle\left\langle v_{-11}^{-}\right|\right)+ \\
& \lambda_{12}^{-}\left(\left|v_{12}^{-}\right\rangle\left\langle v_{12}^{-}|+| v_{-12}^{-}\right\rangle\left\langle v_{-12}^{-}\right|\right)+ \\
& \lambda_{01}^{+}\left|v_{01}^{+}\right\rangle\left\langle v_{01}^{+}\left|+\lambda_{02}^{+}\right| v_{02}^{+}\right\rangle\left\langle v_{02}^{+}\right|+ \\
& \lambda_{03}^{+}\left|v_{03}^{+}\right\rangle\left\langle v_{03}^{+}\right|+ \\
& \lambda_{01}^{-}\left|v_{01}^{-}\right\rangle\left\langle v_{01}^{-}\left|+\lambda_{02}^{-}\right| v_{02}^{-}\right\rangle\left\langle v_{02}^{-}\right| .
\end{aligned}
$$

In the following we shall assume for convenience that the ground state energy of the local interaction $h_{i j}$ is zero, i.e. at least one $\lambda$ is equal to zero. The most general Hamiltonian consistent with the symmetry requirements then has 21 free parameters (seven $a_{j}$ and $15 \lambda$-parameter, one of which is used to fix the local ground state energy), including one trivial scale factor. 
Now the full Hamiltonian (11) is positive-semidefinite since it is the sum of positive-semidefinite operators $h_{i j}$. Therefore its ground state energy $E_{0}$ satisfies $E_{0} \geq 0$. By definition an optimum ground state $\left|\psi_{0}\right\rangle$ has $E_{0}=0.24$ It can be characterized by the condition

$$
h_{i j}\left|\psi_{0}\right\rangle=0 \text { for all }\langle i, j\rangle \quad \Longleftrightarrow \mathcal{H}\left|\psi_{0}\right\rangle=0,
$$

which means that $\left|\psi_{0}\right\rangle$ is simultaneously ground state of all local interactions $h_{i j}$. Therefore finite-size corrections for the ground state energy vanish exactly for all system sizes.

The simplest examples for OGS are tensor product states, e.g. the fully-polarized ferromagnet

$$
\left|\psi_{\mathrm{ferro}}\right\rangle=\prod_{i}|2\rangle_{i}
$$

The corresponding local ground state is $\left|v_{4}\right\rangle=|2,2\rangle$ (see Table 1). It will become a global ground state if one chooses all interaction parameters $\lambda_{\mu n}^{p}$ in (3) as positive except for $\lambda_{4}=0$. The parameters $a_{1}, \ldots, a_{7}$ are arbitrary.

Here we are interested in more complex (antiferromagnetic) ground states that are not given by simple tensor products. We shall now consider a hexagonal lattice with periodic boundary conditions. The specific implementation is depicted in Fig. 1 which also shows the numbering scheme for the sites. The hexagonal lattice is bipartite with sublattices $\mathfrak{A}, \mathfrak{B}$.

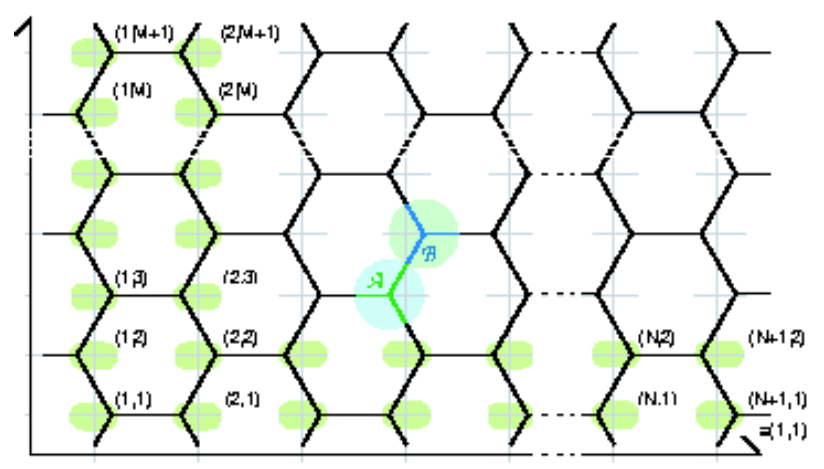

FIG. 1: Numbering scheme for the sites of the hexagonal lattice with sublattices $\mathfrak{A}$ and $\mathfrak{B}$.

\section{Vertex State Model approach}

On the hexagonal lattice there are two types of sites, belonging to the two different sublattices $\mathfrak{A}, \mathfrak{B}$ (see Fig. (1). Correspondingly two types of vertices can be distinguished to which we assign quantum states

$$
\begin{aligned}
& \underline{\mu_{\boldsymbol{\downarrow}}} \hat{\boldsymbol{\mu}} \hat{\boldsymbol{\mu}}=\alpha_{\mu_{1}, \mu_{2}, \mu_{3}}^{1}\left|S_{i}^{z}\left(\mu_{1}, \mu_{2}, \mu_{3}\right)\right\rangle, \quad \text { for all } i \in \mathfrak{A} \text {, } \\
& \sum_{\mu_{\mathbf{1}}}^{\boldsymbol{\mu}_{\mathbf{L}}} \boldsymbol{\mu}_{\mathbf{3}} \hat{=} \alpha_{\mu_{1}, \mu_{2}, \mu_{3}}^{2}\left|S_{i}^{z}\left(\mu_{1}, \mu_{2}, \mu_{3}\right)\right\rangle, \quad \text { for all } i \in \mathfrak{B} \text {, }
\end{aligned}
$$

i.e. the state vector $\left|S_{i}^{z}\right\rangle$ is determined by the values $\mu_{\alpha}$ ( $\alpha=1,2,3)$ of the bond variables belonging to vertex $i$. In the following, $\mu_{\alpha}$ is a 2 -state variable that can be depicted graphically as an arrow pointing in or out of the lattice site.

This general scheme of identifying vertices and quantum states is rather natural. More specifically in the following we use the identification

$$
S_{i}^{z}=n_{i}+\frac{1}{2} \quad \text { or } \quad S_{i}^{z}=n_{i}-\frac{1}{2}
$$

with

$$
n_{i}:=\frac{1}{2}\left(n_{i}^{(+)}-n_{i}^{(-)}\right)
$$

where $n_{i}^{(+)}$and $n_{i}^{(-)}$are the number of arrows pointing out of and into vertex $i$, respectively. Therefore all quantum states generated this way are $S^{z}$-eigenstates of the local spin-2 Hilbert space (see eq. (2)).

There is still some freedom in the choice of the sign in the additive constant eq. (8). Since here we are interested in ground states that are not macroscopically degenerate it will be fixed for each sublattice (see Sec. IIII). For the same reason we will not consider bond variables $\mu_{\alpha}$ that can take more than two states ${ }^{25}$ which is possible in principle. The prefactors $\alpha_{\mu_{1}, \mu_{2}, \mu_{3}}^{i}$ are not determined by this method and can be chosen in a suitable way restricted only by the required symmetries.

In the next step we define quantum states of the full lattice from the single-site states introduced above. As in the partition function of a classical vertex model the bond variables are summed out. For two sites this implies

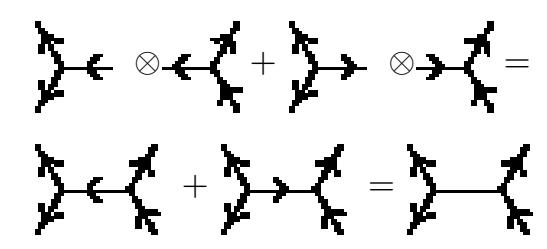

or, explicitly,

$$
\alpha_{1}^{1} \alpha_{2}^{2}|1,1\rangle+\alpha_{2}^{1} \alpha_{2}^{2}|2,1\rangle=\left|\psi^{1,2}\right\rangle .
$$

Here the only difference is replacing the product of Boltzmann weights by a tensor product in spin space. This procedure is generalized to any number of sites in a 
straightforward way to construct a quantum state on the hexagonal lattice consisting of local spin-2 variables:

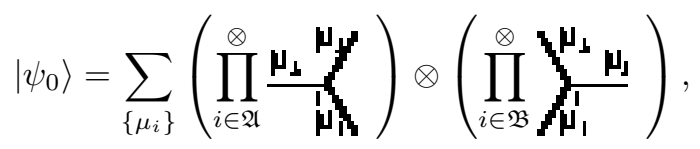

where the black vertices have to be taken for $i \in \mathfrak{A}$ and the grey ones for $i \in \mathfrak{B}$.

So far this procedure is independent of the Hamiltonian under consideration, except for the choice of the local Hilbert space. In the final step we now have to specify the free parameters in the local interaction (3) and the wavefunction (6), (7) in such a way that the quantum state (12) becomes (i) an eigenstate and (ii) a ground state of the Hamiltonian (11). This can be achieved by imposing the optimum ground state condition

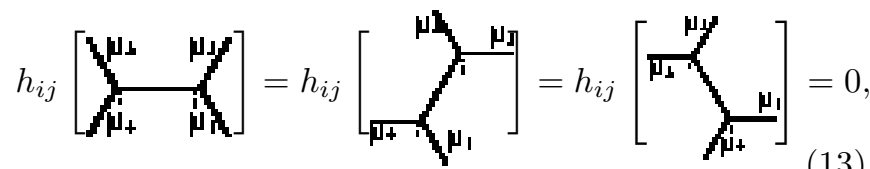

for any pair of nearest neighbors $\langle i, j\rangle$ and all values of $\mu_{\alpha}$ for $\alpha=1,2,3,4$. Since $h_{i j}$ is positive-semidefinite this means that the corresponding 2-spin states (10) are ground states of the local interaction. Therefore, by construction, the global ground state (12) contains only local ground states and thus the optimum ground state condition $\mathcal{H}\left|\psi_{0}\right\rangle=0$ is fulfilled.

\section{GROUND STATES}

In the following we will discuss explicit results for ground state wave functions. By specifying the sign of additive constant in (8) we find two different types of states.

\section{A. The Weak Antiferromagnet}

First consider the case where the additive constant in (8) is fixed to $+\frac{1}{2}$ for sublattice $\mathfrak{A}$ and to $-\frac{1}{2}$ for sublattice $\mathfrak{B}$, e.g. $\rightarrow-\hat{y} \hat{=} \alpha_{1}|1\rangle$ and $\boldsymbol{y}=\alpha_{2}|0\rangle$. The freedom in choosing the sign in (8) can be interpreted as a fourth 2state variable $\mu_{4}$ attached to the site (see (6),(17) ) which is not summed over in the construction of the global state (12). It corresponds to a local spin- $1 / 2$ degree of freedom with magnetization $+\frac{1}{2}$ on sublattice $\mathfrak{A}$ and $-\frac{1}{2}$ on sublattice $\mathfrak{B}$. In this sense we can view the construction described above as starting from a Néel-type reference state with sublattice magnetization $\frac{1}{2}$ which is modified by taking into account the (local) vertex configurations. Therefore we have the following identification of vertices and local quantum states:

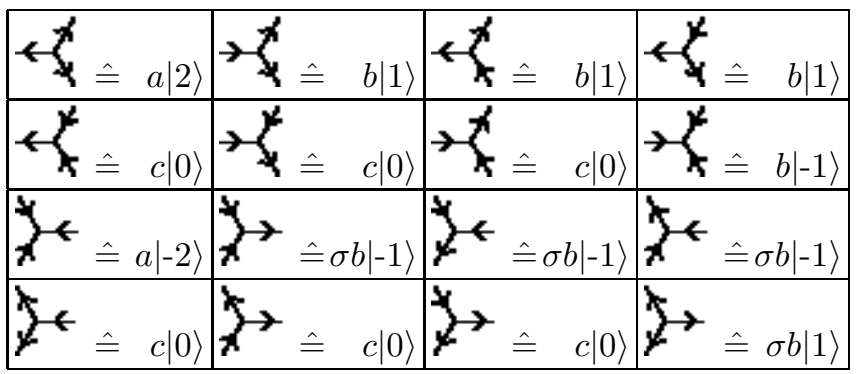

They contain three real parameters $a, b, c \in \mathbb{R}$ and one discrete parameter $\sigma= \pm 1$. One of the real parameters can always be absorbed into the spectral parameters $\lambda$ in (3). This choice of parameters is the most general one allowed by symmetry requirements.

Summing out an inner bond variable leads to nine 2spin states which become local ground states in the following:

$$
\begin{array}{r}
a c|2,0\rangle+\sigma b^{2}|1,1\rangle \\
a c|0,-2\rangle+\sigma b^{2}|-1,-1\rangle \\
\sigma a b|2,-1\rangle+b c|1,0\rangle \\
a b|1,-2\rangle+b c \sigma|0,-1\rangle \\
b c|1,0\rangle_{\sigma} \\
b c|-1,0\rangle_{\sigma} \\
a^{2}|-2,2\rangle+\sigma b^{2}|-1,1\rangle \\
\sigma b^{2}|-1,1\rangle+c^{2}|0,0\rangle \\
\sigma b^{2}|1,-1\rangle+c^{2}|0,0\rangle
\end{array}
$$

As the system should be invariant under time reversal the spin-flipped reference state, which corresponds to an exchange of the sublattices, should also lead to a ground state. This second ground state can be constructed by the following identification of vertices and local states:

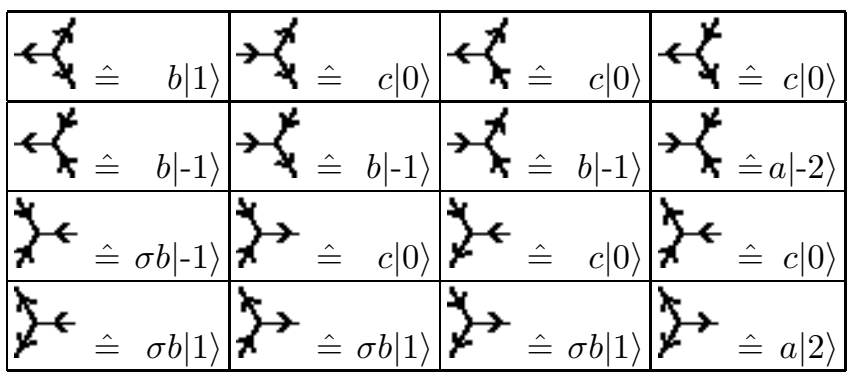

As a result the following five additional local ground states are obtained:

$$
\begin{array}{r}
a c|0,2\rangle+\sigma b^{2}|1,1\rangle \\
a c|-2,0\rangle+\sigma b^{2}|-1,-1\rangle \\
a^{2}|2,-2\rangle+\sigma b^{2}|1,-1\rangle \\
\sigma a b|-1,2\rangle+b c|0,1\rangle \\
a b|-2,1\rangle+b c \sigma|-1,0\rangle .
\end{array}
$$


The total number of local ground states is therefore 14 . The following conditions must be satisfied:

$\lambda_{2}^{-}=\lambda_{22}^{+}=\lambda_{11}^{\sigma}=\lambda_{12}^{+}=\lambda_{12}^{-}=\lambda_{02}^{+}=\lambda_{03}^{+}=\lambda_{01}^{-}=\lambda_{02}^{-}=0$

and

$$
\lambda_{4}, \lambda_{3}^{+}, \lambda_{3}^{-}, \lambda_{21}^{+}, \lambda_{11}^{-\sigma}, \lambda_{01}^{+}>0,
$$

where $\sigma$ is the discrete parameter appearing in the identification of vertices with quantum states. The equations (16) guarantee that the states are eigenstates whereas the inequalities (17) are needed to make them ground states.

The global quantum state (12) is the twofold degenerate ground state for the Hamiltonian (11), (3) satisfying above conditions (16), (17). The degeneracy is exact for all lattice sizes. We do not expect the existence of a phase transition within the parameter region where the optimum ground state is realized.

The global ground state is effectively controlled by two continuous parameters $\frac{a}{c}, \frac{b}{c}$ and one discrete one $\sigma \pm 1$. The global Hamiltonian can be tuned by a parameter manifold of eight continuous (namely the six parameters in (17) and $a / c, b / c$ ) and one discrete parameter $\sigma$ with still a free choice of the scale. By construction, the ground state is antiferromagnetically ordered. From the reference state it inherits a Néel-type order. The sublattice magnetizations $\mathfrak{m}_{\mathfrak{A}}=\left\langle S_{i}^{z}\right\rangle$ for $i \in \mathfrak{A}$ and $\mathfrak{m}_{\mathfrak{B}}=\left\langle S_{i}^{z}\right\rangle$ for $i \in \mathfrak{B}$ depend on the parameters $\frac{a}{c}, \frac{b}{c}$ and $\sigma= \pm 1$ and satisfy

$$
\mathfrak{m}:=\mathfrak{m}_{\mathfrak{A}}=-\mathfrak{m}_{\mathfrak{B}} \quad \text { with } \quad 0 \leq \mathfrak{m} \leq 2 .
$$

Therefore in general the sublattices are not fully polarized $(\mathfrak{m}<2)$ and we call the state a weak antiferromagnet. For $|a| \rightarrow \infty$ one can see from (14) that the local and global ground states are the Néel-states with $\mathfrak{m}=2$. On the other hand, for $|c| \rightarrow \infty$ the ground states becomes $|0 \cdots 0\rangle$ and has $\mathfrak{m}=0$.

\section{B. The Weak Ferromagnet}

It is also possible to start from a different reference state by chosing the same sign for both sublattices in (8). This state is ferromagnetically ordered with magnetization $\frac{1}{2}$. Using the same conventions as above, we obtain the following identification of vertices with local quantum states:

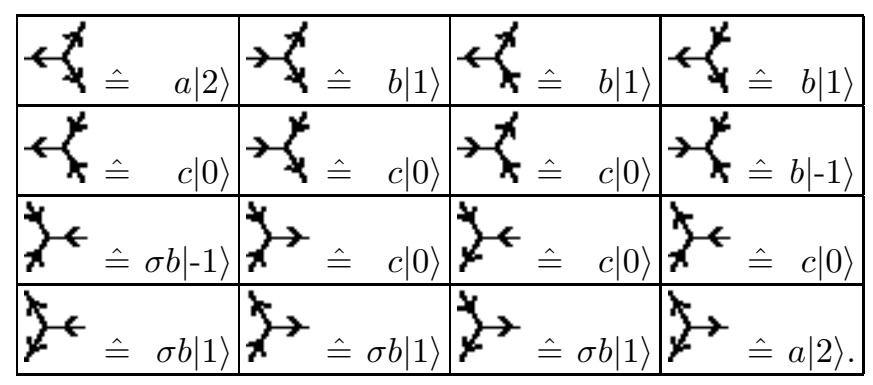

These vertices lead to nine local 2-spin states:

$$
\begin{array}{r}
a b|2,1\rangle_{\sigma} \\
a c|2,0\rangle+\sigma b^{2}|1,1\rangle \\
a c|0,2\rangle+\sigma b^{2}|1,1\rangle \\
\sigma a b|2,-1\rangle+b c|1,0\rangle \\
a b|-1,2\rangle+\sigma b c|0,1\rangle \\
b c|1,0\rangle_{\sigma} \\
c^{2}|0,0\rangle+\sigma b^{2}|-1,1\rangle \\
c^{2}|0,0\rangle+\sigma b^{2}|1,-1\rangle \\
b c|-1,0\rangle_{\sigma},
\end{array}
$$

where $a, b, c$ are real and $\sigma= \pm 1$ is a discrete parameter.

Again the global ground state is obtained by summing over all inner bond variables. The spin-flipped reference state with magnetization $-\frac{1}{2}$ will also lead to a ground state if the following additional vertices are used:

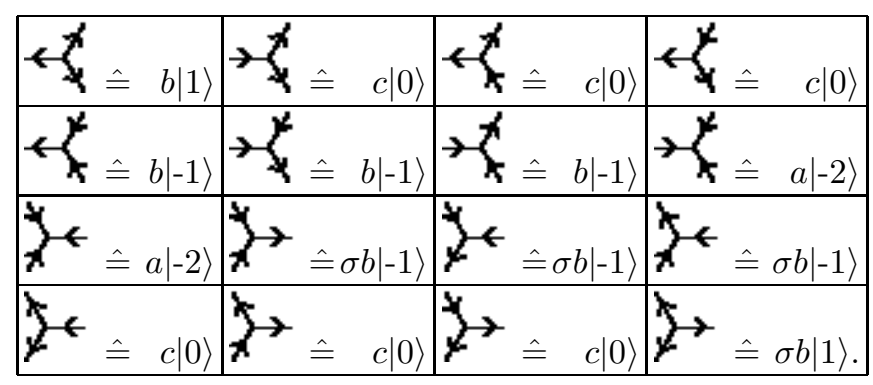

These vertices generate five additional 2-spin states:

$$
\begin{array}{r}
a b|-2,-1\rangle_{\sigma} \\
a c|-2,0\rangle+\sigma b^{2}|-1,-1\rangle \\
a c|0,-2\rangle+\sigma b^{2}|-1,-1\rangle \\
\sigma a b|-2,1\rangle+b c|-1,0\rangle \\
a b|1,-2\rangle+\sigma b c|0,-1\rangle .
\end{array}
$$

The 14 states (19) and (20) are local ground states for

$$
\lambda_{3}^{\sigma}=\lambda_{21}^{+}=\lambda_{2}^{-}=\lambda_{11}^{-}=\lambda_{12}^{\sigma}=\lambda_{03}^{+}=\lambda_{02}^{-}=\lambda_{01}^{-}=0
$$

and

$$
\lambda_{4}, \lambda_{3}^{-\sigma}, \lambda_{22}^{+}, \lambda_{12}^{-\sigma}, \lambda_{01}^{+}, \lambda_{02}^{+}, \lambda_{01}^{-}>0 .
$$

Again, the corresponding global states (12) are controlled by two continuous parameters $\frac{a}{c}, \frac{b}{c}$ and one discrete one $\sigma \pm 1$. The freedom of chosing the reference state such that its magnetization is $+\frac{1}{2}$ or $-\frac{1}{2}$ implies that a priori the ground state is exactly twofold degenerate.

The global state generically has sublattice magnetizations $-1 \leq \mathfrak{m}_{\mathfrak{B}} \leq \frac{1}{2} \leq \mathfrak{m}_{\mathfrak{A}} \leq 2$ or $1 \geq \mathfrak{m}_{\mathfrak{B}} \geq \frac{1}{2} \geq \mathfrak{m}_{\mathfrak{A}} \geq$ -22 . It is convenient to introduce the order parameter $\psi=\mathfrak{m}-\frac{1}{2}$ which is the magnetization relative to the reference state (with additive constant $+1 / 2$ ). Then we have

$$
\psi=\psi_{\mathfrak{A}}=-\psi_{\mathfrak{B}} \quad \text { with } \quad 0 \leq \psi \leq \frac{3}{2}
$$


Note that the magnetization $\mathfrak{m}_{\mathfrak{B}}$ on sublattice $\mathfrak{B}$ can indeed become negative so that the global ground state exhibits an antiferromagnetic ordering.

Most interestingly the structure of the ground state suggests the occurrence of a quantum phase transition in the solvable subspace (21), (22) of the weakly ferromagnetic ground state. If the product $a b$ of the parameters controlling the local ground states becomes large compared to the other coefficients in (19), the dominating 2spin states contributing to the global state are $a b|2,1\rangle_{\sigma}$, $\sigma a b|2,-1\rangle$ and $a b|-1,2\rangle$ (and its spin-flipped counterparts). However, the only global states compatible with periodic boundary conditions that can be constructed from these local states are product states having $|2\rangle$ on sublattice $\mathfrak{A}$ and $|-1\rangle$ on sublattice $\mathfrak{B}$, or vice versa. This implies a fourfold degenerate ground state with sublattice symmetry in the limit $a b \rightarrow \infty$. In this limit the order parameter becomes $\psi=\frac{3}{2}$. On the other hand, for small $a b$ the ground state is only twofold degenerate. Thus we can expect a quantum phase transition at a critical value $(a b)_{c}$ from a fourfold degenerate state to a twofold degenerate state with vanishing order parameter $\psi=0$, i.e. with non-broken sublattice symmetry. The determination of correlation functions requires studying a two-dimensional classical vertex model (see Sec. IV). Preliminary results ${ }^{22}$ indeed show strong evidence for a phase transition line. Numerical results for a representative point indicate a continuous transition.

\section{CONCLUSIONS}

In this paper we have solved a large class of spin-2 quantum spin models on the hexagonal lattice. Using the optimum ground state concept realized through the quantum vertex model approach the explicit form of the ground state wave function could be determined. Focussing on weakly degenerate ground states two different types of states existing in different subspaces have been found. In contrast to the generic VBS states $\frac{18,23}{}$, coordination number $z$ and spin $S$ are not related by $S=z / 2$. Some properties of the states that we have constructed can be understood by interpreting the spin $S=2$ as symmetrization of a spin- $3 / 2$ and a spin- $1 / 2$. For the spin $S=3 / 2$ OGS have been constructed previously 15 . In addition we now have a spin- $1 / 2$ degree of freedom at each site that allows to define different "reference states".

The weak antiferromagnet has a twofold degenerate ground state characterized by a sublattice magnetization $\mathfrak{m}_{\mathfrak{A}}=-\mathfrak{m}_{\mathfrak{B}}$ with $0 \leq \mathfrak{m}_{\mathfrak{A}} \leq 2$. It exists in a subspace controlled by eight continuous and one discrete parameter. The weak ferromagnet is conveniently described by the order parameter $\psi=\mathfrak{m}-\frac{1}{2}$ such that $\psi_{\mathfrak{A}}=-\psi_{\mathfrak{B}}$. We have argued that it exhibits a quantum phase transition from a twofold degenerate disordered phase with $\psi=0$ to a fourfold degenerate phase with broken sublattice symmetry and $\psi>0$.

In future work we will investigate the ground state correlations which will help to understand the properties of the quantum phase transition in more detail. As described in 15 expectation values and correlations are determined by the partition function of a classical vertex model on the same lattice. This is a considerable simplification compared to the generic case where e.g. Quantum Monte Carlo calculations require the investigation of a classical model in dimension $d+1$. However, in our case the classical vertex model has four states per bond and is not exactly solvable. Therefore its properties have to be studied numerically using classical Monte Carlo simulations.

We want to emphasize that the construction of quantum states using vertex models is different from the one used in so-called quantum vertex models $\frac{19}{}$. There the graphical representation of the states in terms of closed loops is used to define the full dynamics of a quantum model, i.e. the full Hamiltonian. In our case the vertex model is only used to specify the ground state. The identification of vertex and quantum states does not extend to excitations. This offers a larger flexibility of the approach.

\section{Acknowledgments}

This work has been performed within the research program SFB 608 of the Deutsche Forschungsgemeinschaft.
* maa@thp.uni-koeln.de

$\dagger$ as@thp.uni-koeln.de

zitt@thp.uni-koeln.de

1 D. Arovas and S. Girvin, in Recent Progress in Many-Body Theories, Vol. 3, edited by T. Ainsworth, C. Campbell, B. Clements, and E. Krotschek (Plenum, New York, 1992).

2 A. Klümper, A. Schadschneider, and J. Zittartz, Europhys. Lett. 24, 293 (1993).

3 J. de Boer and A. Schadschneider, Phys. Rev. Lett. 75, 4298 (1995).

${ }^{4}$ H. Niggemann and J. Zittartz, Z. Phys B 101, 289 (1996).

5 A. Klümper, A. Schadschneider, and J. Zittartz, J. Phys.
A 24, L955 (1991).

${ }^{6}$ A. Klümper, A. Schadschneider, and J. Zittartz, Z. Phys. B 87, 281 (1992).

7 M. Fannes, B. Nachtergaele, and R. Werner, Commun. Math. Phys. 144, 443 (1992).

8 M. A. Ahrens, A. Schadschneider, and J. Zittartz, Europhys. Lett. 59, 889 (2002).

9 S. R. White, Phys. Rev. Lett. 69, 2863 (1992).

10 I. Peschel, W. Wang, M. Kaulke, and K. Hallberg, eds., Density Matrix Renormalisation. A New Numerical Method in Physics, vol. 528 of Lecture Notes in Physics (Springer, Berlin, 1998). 
11 U. Schollwöck, Rev. Mod. Phys. p. in press (2005).

12 S. Östlund and S. Rommer, Phys. Rev. Lett. 75, 3537 (1995).

13 S. Rommer and S. Östlund, Phys. Rev. B 55, 2164 (1997).

14 J. Dukelsky, M. Martin-Delgado, T. Nishino, and G. Sierra, Europhys. Lett. 43, 457 (1998).

15 H. Niggemann, A. Klümper, and J. Zittartz, Z. Phys B 104, 103 (1997).

16 R. J. Baxter, Exactly Solved Models in Statistical Mechanics (Academic Press, London, 1982).

17 H. Niggemann, A. Klümper, and J. Zittartz, Eur. Phys. J. B 13, 15 (2000).

18 I. Affleck, T. Kennedy, E. H. Lieb, and T. Tasaki, Commun. Math. Phys. 115, 477 (1988).

19 E. Ardonne, P. Fendley, and E. Fradkin, Annals Phys. 310,
493 (2004)

20 D. Rokhsar and S. Kivelson, Phys. Rev. Lett. 86, 2376 (1988).

21 E. Fradkin, D. Huse, R. Moessner, V. Oganesyan, and S. Sondhi, Phys. Rev. B 69, 224415 (2004).

22 M. A. Ahrens, A. Schadschneider, and J. Zittartz (2004), in preparation.

23 I. Affleck, T. Kennedy, E. H. Lieb, and T. Tasaki, Phys. Rev. Lett. 59, 799 (1987).

${ }^{24}$ For the case where the local ground state energy is $e_{0} \neq 0$, the global ground state energy scales with the number $N$ of bonds as $E_{0}=N e_{0}$.

251 -state bond variables $\mu_{\alpha}$ lead to simple tensor products only. 
$\mu_{4}{ }^{\mu}$ 
$\sum_{N_{1}}^{\mu_{N_{1}}}$ 


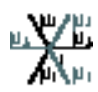




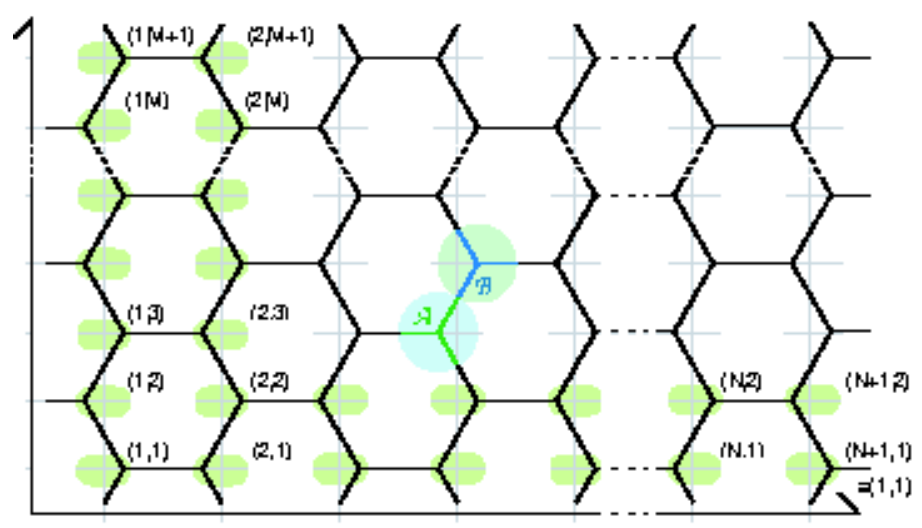


$\xi+x$ 


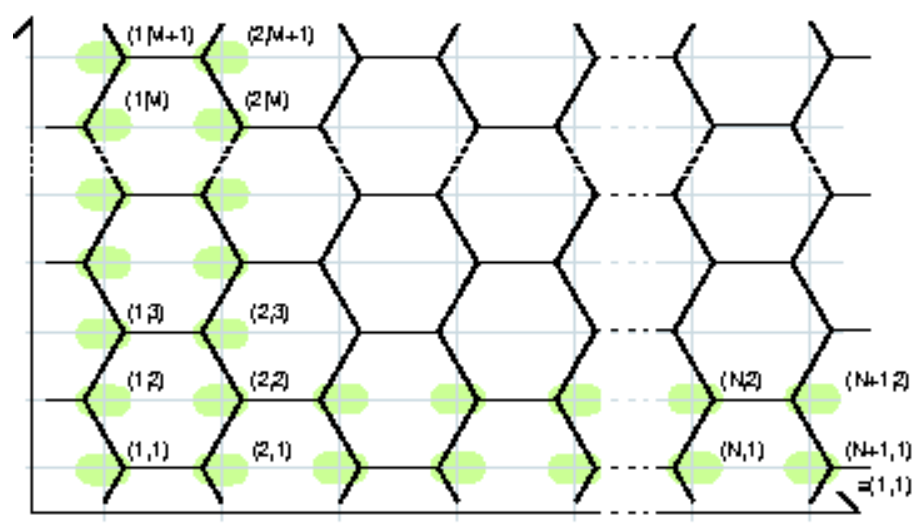


$\xi \rightarrow x$ 
$5 x$ 


$$
\sum_{N_{+}}^{N y}
$$


$\sum_{\mu_{1}}^{\mu_{\mu}}$ 
w... 